УДК 517.5

\author{
E. A. Sevost'Yanov
}

\title{
ISOLATED SINGULARITIES OF MAPPINGS WITH THE INVERSE POLETSKY INEQUALITY
}

\begin{abstract}
E. A. Sevost'yanov Isolated singularities of mappings with the inverse Poletsky inequality, Mat. Stud. 55 (2021), 132-136.

The manuscript is devoted to the study of mappings with finite distortion, which have been actively studied recently. We consider mappings satisfying the inverse Poletsky inequality, which can have branch points. Note that mappings with the reverse Poletsky inequality include the classes of conformal, quasiconformal, and quasiregular mappings. The subject of this article is the question of removability an isolated singularity of a mapping. The main result is as follows. Suppose that $f$ is an open discrete mapping between domains of a Euclidean $n$-dimensional space satisfying the inverse Poletsky inequality with some integrable majorant $Q$. If the cluster set of $f$ at some isolated boundary point $x_{0}$ is a subset of the boundary of the image of the domain, and, in addition, the function $Q$ is integrable, then $f$ has a continuous extension to $x_{0}$. Moreover, if $f$ is finite at $x_{0}$, then $f$ is logarithmic Hölder continuous at $x_{0}$ with the exponent $1 / n$.
\end{abstract}

1. Introduction. In our joint paper [1], we obtained a continuous extension of homeomorphisms, the inverses of which satisfy the weight Poletsky inequality, to an isolated boundary point (see Theorem 5.1). The main purpose of this manuscript is to transfer the specified result to mappings with branching. More precisely, we consider open discrete mappings between two domains of the extended Euclidean space and assume that they satisfy some weight estimate of the distortion of the modulus of families of paths with integrable majorant. Note that the studies of this paper are in the context of studying mappings with bounded and finite distortion (see, e.g., [2]-[8]). The conditions concerning distortion of modulus of families of paths are well known in the theory of quasiconformal mappings and their generalizations (see, for example, [3, Theorem 3.2], [5, Theorem 8.5] and [7, Theorem 6.7.II]).

Let us turn to the definitions. Let $y_{0} \in \mathbb{R}^{n}, 0<r_{1}<r_{2}<\infty$ and

$$
\begin{gathered}
A\left(y_{0}, r_{1}, r_{2}\right)=\left\{y \in \mathbb{R}^{n}: r_{1}<\left|y-y_{0}\right|<r_{2}\right\}, \\
B\left(y_{0}, r\right)=\left\{y \in \mathbb{R}^{n}:\left|y-y_{0}\right|<r\right\}, S\left(y_{0}, r\right)=\left\{y \in \mathbb{R}^{n}:\left|y-y_{0}\right|=r\right\} .
\end{gathered}
$$

Set $\overline{\mathbb{R}^{n}}:=\mathbb{R}^{n} \cup\{\infty\}$. Given sets $E, F \subset \overline{\mathbb{R}^{n}}$ and a domain $D \subset \mathbb{R}^{n}$ denote by $\Gamma(E, F, D)$ the family of all paths $\gamma:[a, b] \rightarrow \overline{\mathbb{R}^{n}}$ such that $\gamma(a) \in E, \gamma(b) \in F$ and $\gamma(t) \in D$ for $t \in[a, b]$. Given a domain $D \subset \mathbb{R}^{n}$, or $D \subset \overline{\mathbb{R}^{n}}$, a mapping $f: D \rightarrow \mathbb{R}^{n}$ is an arbitrary continuous transformation $x=\left(x_{1}, \ldots, x_{n}\right) \mapsto f(x)=\left(f_{1}(x), \ldots, f_{n}(x)\right)$. Let $f: D \rightarrow \mathbb{R}^{n}$, let $y_{0} \in f(D)$

2010 Mathematics Subject Classification: 30C65, 31A15, 30C62.

Keywords: quasiconformal mappings; mappings with bounded and finite distortion; equicontinuity; moduli of families of paths.

doi:10.30970/ms.55.2.132-136

(C) E. A. Sevost'yanov, 2021 
and let $0<r_{1}<r_{2}<d_{0}=\sup _{y \in f(D)}\left|y-y_{0}\right|$. Now, we denote by $\Gamma_{f}\left(y_{0}, r_{1}, r_{2}\right)$ the family of all paths $\gamma$ in $D$ such that $f(\gamma) \in \Gamma\left(S\left(y_{0}, r_{1}\right), S\left(y_{0}, r_{2}\right), A\left(y_{0}, r_{1}, r_{2}\right)\right)$. Everywhere below, $M(\Gamma)$ denotes the modulus of a family $\Gamma$ of paths $\gamma$ in $\mathbb{R}^{n}$, the definition and basic properties of which we assume to be known (see, for example, [8, section 6]). Let $Q: \mathbb{R}^{n} \rightarrow[0, \infty]$ be a Lebesgue measurable function such that $Q(x) \equiv 0$ for $\mathbb{R}^{n} \backslash D$. We will say that $f$ satisfies the inverse Poletsky inequality at a point $y_{0} \in f(D)$, if the relation

$$
M\left(\Gamma_{f}\left(y_{0}, r_{1}, r_{2}\right)\right) \leqslant \int_{f(D) \cap A\left(y_{0}, r_{1}, r_{2}\right)} Q(y) \cdot \eta^{n}\left(\left|y-y_{0}\right|\right) d m(y)
$$

holds for any Lebesgue measurable function $\eta:\left(r_{1}, r_{2}\right) \rightarrow[0, \infty]$ such that

$$
\int_{r_{1}}^{r_{2}} \eta(r) d r \geqslant 1 .
$$

Note that the inequalities (1) are well known in the theory of quasiregular mappings and hold for $Q=N(f, D) \cdot K$, where $N(f, D)$ is the maximal multiplicity of $f$ in $D$, and $K \geqslant 1$ is some constant that may be calculated in the following way: $K=\operatorname{ess} \sup K_{O}(x, f), K_{O}(x, f)=$ $\left\|f^{\prime}(x)\right\|^{n} /|J(x, f)|$ for $J(x, f) \neq 0 ; K_{O}(x, f)=1$ for $f^{\prime}(x)=0$, and $K_{O}(x, f)=\infty$ for $f^{\prime}(x) \neq 0$, where $J(x, f)=0$ (see, e.g., [3, Theorem 3.2] or [7, Theorem 6.7.II]). A mapping $f: D \rightarrow \mathbb{R}^{n}$ is called a discrete, if the pre-image $\left\{f^{-1}(y)\right\}$ consists of isolated points for any $y \in \mathbb{R}^{n}$, and an open, if $f(U)$ is open for any open set $U \subset D$. As usual, we put

$$
C(x, f):=\left\{y \in \overline{\mathbb{R}^{n}}: \exists x_{k} \in D: x_{k} \rightarrow x, f\left(x_{k}\right) \rightarrow y, k \rightarrow \infty\right\} .
$$

Hereinafter, the boundary $\partial D$ and the closure $\bar{D}$ are understood in the topology of the extended Euclidean space $\overline{\mathbb{R}^{n}}$. The following statement holds.

Theorem 1. Let $D$ and $D^{\prime}$ be domains in $\overline{\mathbb{R}^{n}}, n \geqslant 2, x_{0} \in D$, and let $f$ be an open and discrete mapping of $D \backslash\left\{x_{0}\right\}$ onto $D^{\prime}$, such that the relation (1) holds at least one finite point $y_{0} \in C\left(x_{0}, f\right)$. Let $C\left(x_{0}, f\right) \subset \partial D^{\prime}$. If $Q \in L^{1}\left(D^{\prime}\right)$, then $f$ has a continuous extension $f: D \rightarrow \overline{D^{\prime}}$. Moreover, if $x_{0} \neq \infty \neq f\left(x_{0}\right)$, then

$$
\left|f(x)-f\left(x_{0}\right)\right| \leqslant \frac{C_{n} \cdot\left(\|Q\|_{1}\right)^{1 / n}}{\log ^{1 / n}\left(1+\frac{r_{0}}{\left|x-x_{0}\right|}\right)}
$$

for any $0<2 r_{0}<\operatorname{dist}\left(x_{0}, \partial D\right)$ and every $x \in B\left(x_{0}, r_{0}\right)$, where $\|Q\|_{1}$ is the norm of the function $Q$ in $L^{1}\left(D^{\prime}\right)$.

2. Proof of Theorem 1. Without a loss of generality, we may assume that $x_{0} \neq \infty$. Everywhere further $h(x, y)$ denotes the chordal distance between the points $x, y \in \overline{\mathbb{R}}^{n}$ (see e.g. [8, Definition 12.1]). Due to the discreteness of the mapping $f$, there is $0<\varepsilon_{0}<$ $\operatorname{dist}\left(x_{0}, \partial D\right)$ such that $\infty \notin f\left(S\left(x_{0}, \varepsilon\right)\right)$ (if $\partial D=\varnothing$, we choose any $\varepsilon_{0}>0$ with the condition mentioned above). Put $g:=\left.f\right|_{B\left(x_{0}, \varepsilon_{0}\right) \backslash\left\{x_{0}\right\}}$.

Suppose the opposite, namely, that the mapping $f$ does not have a continuous boundary extension to a point $x_{0}$. Then in the same way the mapping $g$ does not have a continuous boundary extension to the same point. Since the space $\overline{\mathbb{R}^{n}}$ is compact, $C\left(x_{0}, f\right)=C\left(x_{0}, g\right) \neq$ 
$\varnothing$. Then there are $y_{1}, y_{2} \in C\left(x_{0}, f\right), y_{1} \neq y_{2}$, and at least two sequences $x_{m}, x_{m}^{\prime} \in B\left(x_{0}, \varepsilon_{0}\right) \backslash$ $\left\{x_{0}\right\}$ such that $x_{m}, x_{m}^{\prime} \rightarrow x_{0}$ as $m \rightarrow \infty$, and $z_{m}:=g\left(x_{m}\right) \rightarrow y_{1}, z_{m}^{\prime}=g\left(x_{m}^{\prime}\right) \rightarrow y_{2}$ as $m \rightarrow \infty$. We may consider that $y_{1} \neq \infty$.

Let $D_{*}:=f\left(B\left(x_{0}, \varepsilon_{0}\right) \backslash\left\{x_{0}\right\}\right)$. We show that there exists $\varepsilon_{1}>0$ such that

$$
B\left(y_{1}, \varepsilon_{1}\right) \cap f\left(S\left(x_{0}, \varepsilon_{0}\right)\right)=\varnothing .
$$

Observe that $y_{1} \in \partial D_{*}$. Indeed, if $y_{1}$ is an inner point of $D_{*}$, then $y_{1}$ is also inner for $D^{\prime}$, because $D_{*} \subset D^{\prime}$. The latter contradicts the condition $C\left(x_{0}, f\right) \subset \partial D^{\prime}$. Next, since $S\left(x_{0}, \varepsilon_{0}\right)$ is a compact set in $D$, then $f\left(S\left(x_{0}, \varepsilon_{0}\right)\right)$ is compact in $D^{\prime}$, therefore $h\left(f\left(S\left(x_{0}, \varepsilon_{0}\right)\right), y_{1}\right)>\delta>$ 0 , where $h(A, B)=\inf _{x \in A, y \in B} h(x, y)$ is the chordal distance between sets $A, B \subset \overline{\mathbb{R}^{n}}$. Hence

$$
\operatorname{dist}\left(y_{1}, f\left(S\left(x_{0}, \varepsilon_{0}\right)\right)\right)>\delta_{1}>0,
$$

where $\operatorname{dist}(A, B)$ denotes the Euclidean distance between sets $A$ and $B$ in $\mathbb{R}^{n}$. By (5), the relation (4) holds for $\varepsilon_{1}:=\delta_{1}$.

Now we will reason as follows. Let $B_{*}\left(y_{2}, \varepsilon_{2}\right)=B\left(y_{2}, \varepsilon_{2}\right)$ for $y_{2} \neq \infty$ and $B_{*}\left(y_{2}, \varepsilon_{2}\right)=$ $\left\{x \in \overline{\mathbb{R}^{n}}: h(x, \infty)<\varepsilon_{2}\right\}$ for $y_{2}=\infty$. Arguing similarly to the proof of the relation (4), we may show that there is $\varepsilon_{2}>0$ such that $B_{*}\left(y_{2}, \varepsilon_{2}\right) \cap f\left(S\left(x_{0}, \varepsilon_{0}\right)\right)=\varnothing$. Without loss of the

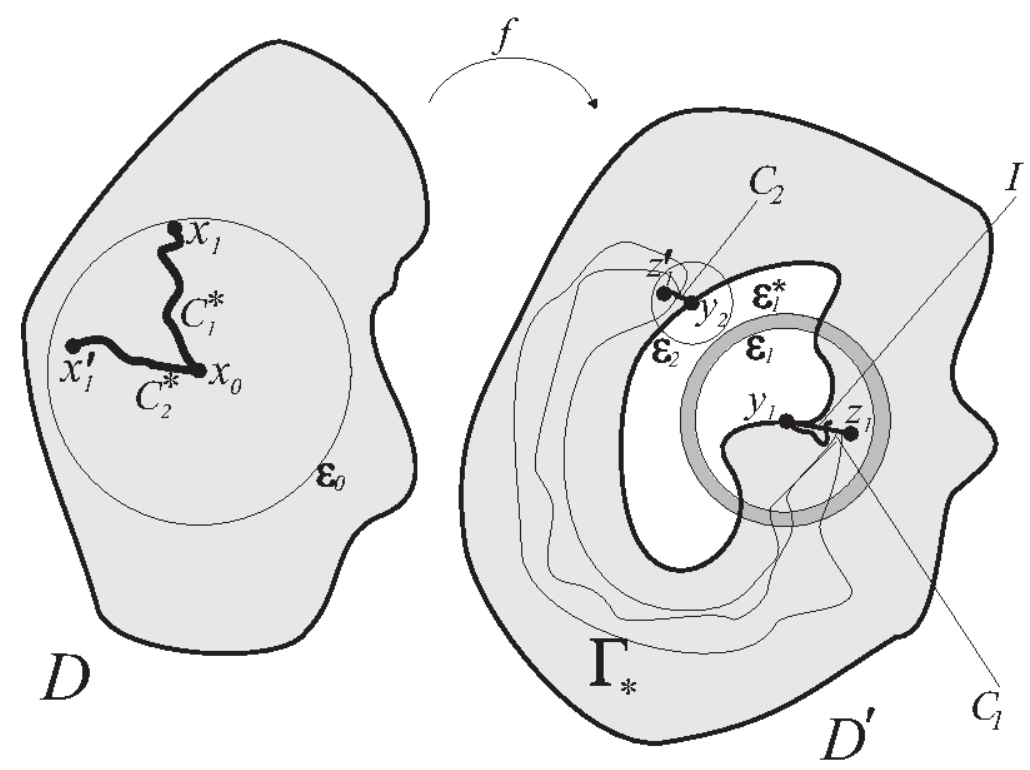

Figure 1: To the proof of Theorem 1

generality, we may assume that $\overline{B\left(y_{1}, \varepsilon_{1}\right)} \cap \overline{B_{*}\left(y_{2}, \varepsilon_{2}\right)}=\varnothing$, in addition, $z_{m} \in B\left(y_{1}, \varepsilon_{1}\right)$ and $z_{m}^{\prime} \in B_{*}\left(y_{2}, \varepsilon_{2}\right)$ (see Figure 1$)$.

Note that, $B\left(y_{1}, \varepsilon_{1}\right)$ is convex, and $B_{*}\left(y_{2}, \varepsilon_{2}\right)$ is linearly path connected. In this case, the points $z_{1}$ and $y_{1}$ may be joined by the segment $I(t)=z_{1}+t\left(y_{1}-z_{1}\right), t \in(0,1)$, which lies entirely in $B\left(y_{1}, \varepsilon_{1}\right)$. Similarly, points $z_{1}^{\prime}$ and $y_{2}$ may be joined by a path $J=J(t), t \in[0,1]$, which lies in the "ball" $B_{*}\left(y_{2}, \varepsilon_{2}\right)$.

Observe that, by the construction, $|I| \cap \partial D_{*} \neq \varnothing \neq|J| \cap \partial D_{*}$. Set $t_{*}:=\sup \left\{t: t \in[0,1], I(t) \in D_{*}\right\}, \quad p_{*}:=\sup \left\{t: t \in[0,1], J(t) \in D_{*}\right\}$. Let $C_{1}:=I_{\left[0, t_{*}\right)}, \quad C_{2}:=J_{\left[0, p_{*}\right)}$.

By [4, Lemma 3.12], $C_{1}$ and $C_{2}$ have maximal $f$-liftings $C_{1}^{*}:\left[0, c_{1}\right) \rightarrow B\left(x_{0}, \varepsilon_{0}\right) \backslash\left\{x_{0}\right\}$ and $C_{2}^{*}:\left[0, c_{2}\right) \rightarrow B\left(x_{0}, \varepsilon_{0}\right) \backslash\left\{x_{0}\right\}$ starting at the points $x_{1}$ and $x_{1}^{\prime}$, respectively. Note that 
the case $C_{1}(t) \rightarrow z_{0}$ as $t \rightarrow c_{1}-0$, where $z_{0} \in B\left(x_{0}, \varepsilon_{0}\right) \backslash\left\{x_{0}\right\}$, is impossible. Indeed, by [4, Lemma 3.12], $c_{1}=t_{*}$ and $I(t) \rightarrow f\left(z_{0}\right) \in D_{*}$, that contradicts the definition of $t_{*}$. Now, by [4, Lemma 3.12]

$$
h\left(C_{1}^{*}(t), \partial\left(B\left(x_{0}, \varepsilon_{0}\right) \backslash\left\{x_{0}\right\}\right)\right) \rightarrow 0, \quad t \rightarrow c_{1}-0 .
$$

We show that the case $h\left(C_{1}^{*}(t), S\left(x_{0}, \varepsilon_{0}\right)\right) \rightarrow 0$ as $t \rightarrow c_{1}-0$ is also impossible. Indeed, otherwise $h\left(C_{1}^{*}\left(t_{k}\right), S\left(x_{0}, \varepsilon_{0}\right)\right) \rightarrow 0$ as $k \rightarrow \infty$ for some sequence $t_{k} \rightarrow c-0$. Due to the compactness of the sphere $S\left(x_{0}, \varepsilon_{0}\right)$ there is a sequence $w_{k} \in S\left(x_{0}, \varepsilon_{0}\right)$ such that

$$
h\left(C_{1}^{*}\left(t_{k}\right), S\left(x_{0}, \varepsilon_{0}\right)\right)=h\left(C_{1}^{*}\left(t_{k}\right), w_{k}\right) .
$$

Again, since the sphere $S\left(x_{0}, \varepsilon_{0}\right)$ is compact, we may assume that $w_{k} \rightarrow w_{0}$ as $k \rightarrow \infty$. Then $C_{1}^{*}\left(t_{k}\right) \rightarrow w_{0}$ as $k \rightarrow \infty$. By the continuity of $f$ in $D$ we obtain that

$$
f\left(C_{1}^{*}\left(t_{k}\right)\right)=C_{1}\left(t_{k}\right) \rightarrow f\left(w_{0}\right) \in f\left(S\left(x_{0}, \varepsilon_{0}\right)\right) \text { as } k \rightarrow \infty .
$$

The latter contradicts the condition (4), because simultaneously $f\left(w_{0}\right) \in f\left(S\left(x_{0}, \varepsilon_{0}\right)\right)$ and $f\left(w_{0}\right) \in|I| \subset B\left(y_{1}, \varepsilon_{1}\right)$. Then, it follows from (6) that,

$$
h\left(C_{1}^{*}(t), x_{0}\right) \rightarrow 0, \quad t \rightarrow c_{1}-0 .
$$

Applying similar considerations to the path $C_{2}^{*}(t)$, we may show that

$$
h\left(C_{2}^{*}(t), x_{0}\right) \rightarrow 0, \quad t \rightarrow c_{2}-0 .
$$

By (7) and (8), and by [8, Theorem 10.12] we obtain that

$$
M\left(\Gamma\left(\left|C_{1}^{*}(t)\right|,\left|C_{2}^{*}(t)\right|, B\left(x_{0}, \varepsilon_{0}\right) \backslash\left\{x_{0}\right\}\right)\right)=\infty .
$$

We show that (9) contradicts the condition (1) at the point $y_{0}=y_{1}$. Since $\overline{B\left(y_{1}, \varepsilon_{1}\right)} \cap$ $\overline{B_{*}\left(y_{2}, \varepsilon_{2}\right)}=\varnothing$, we may found $\varepsilon_{1}^{*}>\varepsilon_{1}$, for which we still have $\overline{B\left(y_{1}, \varepsilon_{1}^{*}\right)} \cap \overline{B_{*}\left(y_{2}, \varepsilon_{2}\right)}=\varnothing$. Let $\Gamma_{*}=\Gamma\left(\left|C_{1}\right|,\left|C_{2}\right|, D_{*}\right)$. Observe that

$$
\Gamma_{*}>\Gamma\left(S\left(y_{1}, \varepsilon_{1}^{*}\right), S\left(y_{1}, \varepsilon_{1}\right), A\left(y_{1}, \varepsilon_{1}, \varepsilon_{1}^{*}\right)\right) .
$$

Indeed, let $\gamma \in \Gamma_{*}, \gamma:[a, b] \rightarrow \mathbb{R}^{n}$. Since $\gamma(a) \in\left|C_{1}\right| \subset B\left(y_{1}, \varepsilon_{1}\right)$ and $\gamma(b) \in\left|C_{2}\right| \subset$ $\overline{\mathbb{R}^{n}} \backslash B\left(y_{1}, \varepsilon_{1}\right)$, by [9, Theorem 1.I.5.46] there is $t_{1} \in(a, b)$ such that $\gamma\left(t_{1}\right) \in S\left(y_{1}, \varepsilon_{1}\right)$. Without loss of generality, we may assume that $\left|\gamma(t)-y_{1}\right|>\varepsilon_{1}$ for $t>t_{1}$. Since $\gamma\left(t_{1}\right) \in B\left(y_{1}, \varepsilon_{1}^{*}\right)$ and $\gamma(b) \in\left|C_{2}\right| \subset \mathbb{R}^{n} \backslash B\left(y_{1}, \varepsilon_{1}^{*}\right)$, by [9, Theorem 1.I.5.46] there is $t_{2} \in\left(t_{1}, b\right)$ such that $\gamma\left(t_{2}\right) \in$ $S\left(y_{1}, \varepsilon_{1}^{*}\right)$. Without loss of generality, we may assume that $\left|\gamma(t)-y_{1}\right|<\varepsilon_{1}^{*}$ for $t_{1}<t<t_{2}$. Therefore, $\left.\gamma\right|_{\left[t_{1}, t_{2}\right]}$ is a subpath of $\gamma$, which belongs to $\Gamma\left(S\left(y_{1}, \varepsilon_{1}^{*}\right), S\left(y_{1}, \varepsilon_{1}\right), A\left(y_{1}, \varepsilon_{1}, \varepsilon_{1}^{*}\right)\right)$. Thus, the relation (10) is proved.

Let us prove that

$$
\Gamma\left(\left|C_{1}^{*}(t)\right|,\left|C_{2}^{*}(t)\right|, B\left(x_{0}, \varepsilon_{0}\right) \backslash\left\{x_{0}\right\}\right)>\Gamma_{f}\left(y_{1}, \varepsilon_{1}, \varepsilon_{1}^{*}\right) .
$$

Indeed, if $\gamma:[a, b] \rightarrow B\left(x_{0}, \varepsilon_{0}\right) \backslash\left\{x_{0}\right\}$ belongs to $\Gamma\left(\left|C_{1}^{*}(t)\right|,\left|C_{2}^{*}(t)\right|, B\left(x_{0}, \varepsilon_{0}\right) \backslash\left\{x_{0}\right\}\right)$, then $f(\gamma)$ belongs to $D_{*}$, in addition, $f(\gamma(a)) \in\left|C_{1}\right|$ and $f(\gamma(b)) \in\left|C_{2}\right|$, i.e., $f(\gamma) \in \Gamma_{*}$. Then, according to the above and by $(10), f(\gamma)$ has a subpath $f(\gamma)^{*}:=\left.f(\gamma)\right|_{\left[t_{1}, t_{2}\right]}, a \leqslant t_{1}<t_{2} \leqslant b$, belonging to $\Gamma\left(S\left(y_{1}, \varepsilon_{1}^{*}\right), S\left(y_{1}, \varepsilon_{1}\right), A\left(y_{1}, \varepsilon_{1}, \varepsilon_{1}^{*}\right)\right)$. Then $\gamma^{*}:=\left.\gamma\right|_{\left[t_{1}, t_{2}\right]}$ is a subpath of $\gamma$ and it belongs to $\Gamma_{f}\left(y_{1}, \varepsilon_{1}, \varepsilon_{1}^{*}\right)$, which was required to prove. Put $\eta(t)= \begin{cases}1 /\left(\varepsilon_{1}^{*}-\varepsilon_{1}\right), & t \in\left[\varepsilon_{1}, \varepsilon_{1}^{*}\right], \\ 0, & t \in \mathbb{R} \backslash\left[\varepsilon_{1}, \varepsilon_{1}^{*}\right] .\end{cases}$ 
Observe that $\eta$ satisfies (2) for $r_{1}=\varepsilon_{1}$ and $r_{2}=\varepsilon_{1}^{*}$. Applying (1) at $y_{1}$, and taking into account the relation (11), we obtain that

$$
M\left(\Gamma\left(\left|C_{1}^{*}(t)\right|,\left|C_{2}^{*}(t)\right|, B\left(x_{0}, \varepsilon_{0}\right) \backslash\left\{x_{0}\right\}\right)\right) \leqslant M\left(\Gamma_{f}\left(y_{1}, \varepsilon_{1}, \varepsilon_{1}^{*}\right)\right) \leqslant\|Q\|_{1} /\left(\varepsilon_{1}^{*}-\varepsilon_{1}\right)^{n}<\infty,
$$

where $\|Q\|_{1}$ denotes $L^{1}$-norm of $Q$ in $D^{\prime}$. This relation and relation (9) contradict each other, which refutes the assumption of the existence of different $y_{1}$ and $y_{2}$ in $C\left(x_{0}, f\right)$.

Finally, if $x_{0} \neq \infty$, we consider a domain $D_{1}:=D \backslash\left\{f^{-1}(\infty)\right\}$. Note that, due to the discreteness of the mapping $f$, the set $\left\{f^{-1}(\infty)\right\}$ is at most countable. Thus, $D_{1}$ is a domain, and the point $x_{0}$ is its inner point. Arguing similarly to the second part of the proof of [10, Theorem 6.4], one can show that the mapping $f: D_{1} \rightarrow \mathbb{R}^{n}$ is also open and discrete. In this case, the relation (3) holds by [11, Theorem 1.1].

\section{REFERENCES}

1. E.A. Sevost'yanov, S.A. Skvortsov, On mappings whose inverse satisfy the Poletsky inequality, Ann. Acad. Scie. Fenn. Math., 45 (2020), 259-277.

2. V. Ya. Gutlyanskii, V. I. Ryazanov, E. Yakubov, The Beltrami equations and prime ends, Ukr. Mat. Visn., 12 (2015), №1, 27--66; transl. J. Math. Sci. (N.Y.), 210 (2015), №1, 22-51.

3. O. Martio, S. Rickman, J. Väisälä, Definitions for quasiregular mappings, Ann. Acad. Sci. Fenn. Ser. A1, 448 (1969), 1-40.

4. O. Martio, S. Rickman, J. Väisälä, Topological and metric properties of quasiregular mappings, Ann. Acad. Sci. Fenn. Ser. A1, 488 (1971), 1-31.

5. O. Martio, V. Ryazanov, U. Srebro, E. Yakubov, Moduli in modern mapping theory, Springer Monographs in Mathematics, Springer, New York etc., 2009.

6. O. Martio, U. Srebro, Periodic quasimeromorphic mappings in $\mathbb{R}^{n}$, J. d'Anal. Math., 28 (1975), №1, 20-40.

7. S. Rickman, Quasiregular mappings, Springer-Verlag, Berlin, 1993.

8. J. Väisälä, Lectures on n-dimensional quasiconformal mappings, Lecture Notes in Math., 229, SpringerVerlag, Berlin etc., 1971.

9. K. Kuratowski, Topology, V.2, Academic Press, New York-London, 1968.

10. E. Sevost'yanov, Towards a theory of removable singularities for maps with unbounded characteristic of quasi-conformity, Izv. Math., 74 (2010), №1, 151-165.

11. E. Sevost'yanov, S. Skvortsov, O. Dovhopiatyi, On nonhomeomorphic mappings with the inverse Poletsky inequality, Ukr. Mat. Visnyk, 17 (2020), №3, 414-436. (in Ukrainian)

Zhytomyr Ivan Franko State University

Zhytomyr, Ukraine

Institute of Applied Mathematics and Mechanics of NAS of Ukraine

Slovyansk, Ukraine

esevostyanov2009@gmail.com

Received 03.01.2021 